that the rank of $H^{i}(K ; J)$ is the sum of the ranks of $H^{i}(X ; J)$ and $H^{i}(K, X ; J)$ so equals

$$
\left(\begin{array}{c}
2 n \\
i
\end{array}\right)+2^{2 n} \text { if } i \text { is even and } 0<i<2 n,
$$

and this completes the proof.

University of Chicago

\title{
A FIXED POINT THEOREM FOR CONTINUOUS MULTI-VALUED TRANSFORMATIONS
}

\section{ROBERT L. PLUNKETT}

1. Introduction. There are several different definitions of continuity for multi-valued transformations in existence in the literature [1]. Each definition is accompanied naturally by the question: which topological spaces $X$ have the property that, for each continuous multi-valued transformation $F$ of $X$ into $X$, there exists an $x \in X$ with $x \in F(x)$ ? This property is abbreviated F.p.p. and the point $x$ such that $x \in F(x)$ is called a fixed point under $F$. In [2], using one brand of continuity, Strother has shown that each closed and bounded interval $I$ of real numbers has the F.p.p., but that the square, $I \times I$, does not have it. Here, the concept of continuity will be the same as that in [2] and we shall answer the question above, restricting the topological spaces to be continuous curves (compact, locally connected, metric continua). More specifically, we shall prove that a continuous curve has the F.p.p. if and only if it is a dendrite [3, p. 88].

We shall employ the following characterization of continuity due to Strother [1]:

A multi-valued transformation $F$ of a space $X$ into a compact Hausdorff space $Y$ is continuous if and only if, for each $x_{0} \in X$, it is true that:

(1) $F\left(x_{0}\right)$ is closed,

(2) $V$ open and containing $F\left(x_{0}\right)$ implies that there exists an open set $U^{\prime}$ containing $x_{0}$ such that, if $x \in U^{\prime}$, then $F(x) \subset V$, and

Presented to the Society, April 16, 1955; received by the editors March 5, 1955. 
(3) $y_{0} \in F\left(x_{0}\right), y_{0} \in V$, and $V$ open imply that there is an open set $U^{\prime \prime}$ containing $x_{0}$ such that, if $x \in U^{\prime \prime}$, then $F(x) \cdot V \neq \varnothing$.

2. Proofs. Each dendrite has a convex metric [4] and, since a dendrite $D$ contains no simple closed curve, each convex metric for $D$ is a convex metric with unique segments. We consider, in the first theorem, that the metric is convex, because the uniqueness of the segments simplifies some of the arguments.

THEOREM 1. Each dendrite has the F.p.p.

Proof. Suppose the statement is false. Then there exists a dendrite $D$ and a continuous multi-valued transformation $F: D \rightarrow D$ without a fixed point. Let $a \in D$ and consider the set $E=\{x \mid x \in D$ and there exists $z \in F(x)$ such that $x$ separates $a$ and $z\}$. We shall show that the initial supposition implies that $E$ is nonempty and that $E+\{a\}$ is closed, which will provide a contradiction.

(i) $E$ is nonempty. Let $z$ be a point of $F(a)$. Then $z \neq a$, since' $a \notin F(a)$. Let $D_{z}$ be the component of $D-\{a\}$ containing $z$. Let $U$ and $V$ be open sets such that $a \in U, z \in V$ and $U \cdot V=\varnothing$. Let $V^{\prime}$ be open and connected and such that $z \in V^{\prime} \subset V \cdot D_{z}$. By the continuity of $F$, there exists an open set $U^{\prime}$, about $a$, such that $x \in U^{\prime}$ implies $F(x) \cdot V^{\prime} \neq \varnothing$. Let $U^{\prime \prime}=U^{\prime} \cdot U$ and choose $x \in U^{\prime \prime} \cdot \operatorname{seg}(a z)$ such that $x \neq a$. (We denote the unique segment from $a$ to $z$ by seg $(a z)$.) Let $z^{\prime} \in F(x) \cdot V^{\prime}$. Then $x$ separates $z$ from $a$, but does not separate $z$ from $z^{\prime}$, since $x \notin V^{\prime}$. Consequently, $x$ separates $z^{\prime}$ from $a$, which shows that $E$ is not empty.

(ii) $E+\{a\}$ is closed. We call this set $E^{\prime}$. Suppose $x$ is a limit point of $E$, different from $a$. Let $\left\{x_{i}\right\}$ be a sequence of points of $E^{\prime}$ such that $x_{i} \neq a$, for each $i$, and $\left\{x_{i}\right\} \rightarrow x$. If this is not an infinite sequence, then clearly $x \in E^{\prime}$. If $\left\{x_{i}\right\}$ is an infinite sequence, let $z_{i}$ be a point, for each $i$, such that $z_{i} \in F\left(x_{i}\right)$ and such that $x_{i}$ separates $a$ from $z_{i}$. If $\left\{z_{i}\right\}$ is infinite, let $z$ be a limit point, select a subsequence converging to $z$ and the corresponding subsequence of $\left\{x_{i}\right\}$. To save notation, suppose $\left\{z_{i}\right\}$ and $\left\{x_{i}\right\}$ denote the new sequences. If $z \notin F(x)$, then, from the characterization of continuity of $F$, there exist open sets $N, M$, and $V$ such that $z \in N, x \in V, F(x) \subset M, N \cdot M=\varnothing$, and $x^{\prime} \in V$ implies $F\left(x^{\prime}\right) \subset M$. Then, if $n$ is sufficiently large, $x_{n} \in V$ and $z_{n} \in N$, a contradiction, since $x_{n} \in V$ implies $z_{n} \in F\left(x_{n}\right) \subset M$. A similar argument provides a contradiction if $\left\{z_{i}\right\}$ is finite. Therefore $z \in F(x)$.

We must also show that $x$ separates $z$ and $a$. By choice, we have $x \neq a$ and, since $z \in F(x), z \neq x$. Also $z \neq a$, for if it were, and $\epsilon=(1 / 3)$ $\cdot \rho(a, x)$, then, for $i$ sufficiently large, $\rho\left(a, z_{i}\right)<\epsilon$ and $\rho\left(x_{i}, x\right)<\epsilon$. Since 
$\rho(a, x) \leqq \rho\left(a, x_{i}\right)+\rho\left(x_{i}, x\right)$, we would then have, for $i$ sufficiently large, $\rho\left(a, x_{i}\right) \geqq 2 \epsilon$ while $\rho\left(a, z_{i}\right)<\epsilon$. Therefore, $x_{i}$ would not separate $a$ and $z_{i}$, a contradiction. Hence, $x \neq a \neq z \neq x$. Now, if $x$ does not separate $a$ and $z$, then $a, z$, and seg $(a z)$ belong to the same component $D_{x}$ of $D-\{x\}$. Let $V$ be a connected neighborhood of $z$ such that $\bar{V} \subset D_{x}$. Let $U$ be a neighborhood of $x$ such that $U \cdot(\bar{V}+\operatorname{seg}(a z))=\varnothing$. For $n$ sufficiently large, $x_{n} \in U$ and $z_{n} \in V$, and it is seen that $x_{n}$ does not separate $a$ from $z_{n}$, a contradiction. Therefore, there exists a $z \in F(x)$ such that $x$ separates $a$ and $z$. Therefore, $x \in E^{\prime}$ and $E^{\prime}$ is closed.

(iii) The contradiction. Since $E^{\prime}$ is closed, there exists a point $y \in E^{\prime}$ such that $\rho(a, y)=\sup \left\{\rho(a, x), x \in E^{\prime}\right\}$. Since $E$ is nonempty, $y \neq a$; i.e., $y \in E$. There exists, then, a point $z \in F(y)$ such that $y$ separates $a$ and $z$. Therefore, $z$ belongs to a component $D_{y}$ of $D-\{y\}$ such that $a \notin D_{y}$. Let $U$ and $V$ be connected open sets such that $y \in U, z \in V$, $U \cdot V=\varnothing$, and $x \in U$ implies $F(x) \cdot V \neq \varnothing$. Let $\bar{x} \in U \cdot(\operatorname{seg}(y z)-\{y\})$ and $\bar{z} \in V \cdot F(\bar{x})$. Then $\bar{x} \in D_{y}$, so $y$ separates $a$ from $\bar{x}$. Therefore, $\rho(a, \bar{x})>\rho(a, y)$. Also $\bar{x}$ separates $a$ and $\bar{z}$; for, if not, then the connected set $K=V+\operatorname{seg}(a \bar{z})+\operatorname{seg}(a y)$ contains $y$ and $z$, but not $\bar{x}$, contradicting the fact that $\bar{x}$ separates $y$ and $z$. Therefore, $\bar{x} \in E$. That $\rho(a, \bar{x})>\rho(a, y)$ contradicts the fact that $\rho(a, y)=\sup \{\rho(a, x)$, $\left.x \in E^{\prime}\right\}$.

Hence the original assumption must be false; i.e., each dendrite $D$ has the F.p.p.

We prove next that each nondegenerate continuous curve which is not a dendrite does not have the F.p.p., by demonstrating for such a space $X$ a continuous multi-valued transformation of $X$ into $X$ which does not have a fixed point. The construction of the transformation is an obvious generalization of a construction in [2] and utilizes the fact that, under these conditions, the space must contain a simple closed curve.

THEOREM 2. If $X$ is a nondegenerate continuous curve which is not a dendrite, then $X$ does not have the F.p.p.

Proof. $X$ contains a simple closed curve $C^{\prime}$, if it satisfies the hypotheses. Let $h$ be a homeomorphism of $C^{\prime}$ onto $C$, the unit circle in the plane with center at the origin. Let the metric $D$ on $C$ be given by: $D(x, y)=$ length of the shortest arc containing $x$ and $y$. Also, since $C^{\prime}$ is an ANR, there exists an open set $W$ containing $C^{\prime}$ and a continuous retraction $r$ : $W$ onto $C^{\prime}$. Let $V$ be an $\epsilon$-neighborhood of $C^{\prime}$ such that $V \subset W$. If $x \in V$, let $f(x)=(1 / \epsilon) \rho\left(x, C^{\prime}\right)$. Then $f$ is continuous and maps $V$ into $[0,1)$. We also define, for $x \in V, A(x)$ to be the arc of $C$ with center at $h r(x)$ and length $2 \pi f(x)$. (If $f(x)=0$, let 
$A(x)$ be the point $h r(x)$.)

Now define a multi-valued transformation $F^{\prime}: X \rightarrow X$ as follows:

$$
F^{\prime}(x)=\left\{\begin{array}{lr}
h^{-1}(A(x)), & \text { if } x \in V, \\
C^{\prime} & \text { if } x \in X-V .
\end{array}\right.
$$

A straight-forward argument will show that $F^{\prime}$ is a continuous multivalued transformation of $X$ onto $C^{\prime}$. Let $R$ be a rotation of $C$ through $\pi$ radians. Define $F: X \rightarrow C^{\prime}$ by $F=h^{-1} R h F^{\prime}$. Now $F$ is a continuous multi-valued transformation, since $h^{-1} R h$ is a homeomorphism of $C^{\prime}$ onto $C^{\prime}$. But $F$ does not have a fixed point. For, if $x \in X-C^{\prime}$, then $F(x) \subset C^{\prime}$, and, if $x \in C^{\prime}$, then $F(x)=h^{-1} R h F^{\prime}(x)=h^{-1} R h(x)$, a point clearly not equal to $x$.

Therefore, $X$ does not have the F.p.p.

3. Conclusions. Putting Theorems 1 and 2 together, we have, therefore, proved:

Theorem 3. A nondegenerate continuous curve has the F.p.p. if and only if it is a dendrite.

If $X$ and $Y$ are continuous curves each containing more than one point, then $X \times Y$ contains a simple closed curve and, hence, is not a dendrite. We obtain thus the following statement, similar to a conclusion in [2]:

THEOREM 4. If $X$ and $Y$ are nondegenerate continuous curves, then $X \times Y$ does not have the F.p.p.

\section{BIBLIOGRAPHY}

1. W. L. Strother, Continuous multi-valued functions, Dissertation, Tulane University, 1951.

2. - On an open question concerning fixed points, Proc. Amer. Math. Soc. vol. 4 (1953) pp. 988-993.

3. G. T. Whyburn, Analytic topology, Amer. Math. Soc. Colloquium Publications, vol. $28,1942$.

4. R. H. Bing, Partitioning continuous curves, Bull. Amer. Math. Soc. vol. 58 (1952) pp. 536-556.

Florida State University 